\title{
Heterosis for Yield and Yield Contributing Characters in Rice (Oryza sativa L.)
}

\author{
K. K. Barhate*, D. N. Borole and P. R. Kore \\ Department of Botany, College of Agriculture, Dhule -424 004, M.S., India \\ *Corresponding author
}

\section{A B S T R A C T}

\begin{tabular}{|l|}
\hline Key w or d s \\
$\begin{array}{l}\text { Self pollinated } \\
\text { crops, breeding, } \\
\text { heterosis, hybrid, } \\
\text { Oryza sativa }\end{array}$ \\
\hline Article Info \\
\hline $\begin{array}{l}\text { Accepted: } \\
\text { 12 May } 2021 \\
\text { Available Online: } \\
\text { 10 June 2021 }\end{array}$ \\
\hline \hline
\end{tabular}

An investigation was taken up to study the magnitude of heterosis for nine characters in rice viz., days to 50 percent flowering, days to maturity, plant height at maturity, productive tillers per plant, tillers per square meter, thousand grain weight, grains per panicle, and yield per plant. The base material for this experiment consisted of $36 \mathrm{~F} 1 \mathrm{~s}$ involving three cytoplasmic male sterile lines (CMS) and twelve diverse restorers in line $\mathrm{x}$ tester mating design along with best varietal check Phule Samruddhi and best hybrid check Sahyadri-4. Hybrids were evaluated in randomized block design (RBD) with three replications. Heterosis over mid parent, better parent and standard checks revealed that experimental hybrids had higher heterotic effect for all the characters under study. Majority of hybrids exhibited negative heterosis for days to 50 per cent flowering, days to maturity. An appreciable amount of heterosis was observed in yield and yield contributing characters. Thus, the hybrids RTN17-A x VDN-1335 for earliness and IR58025-A x VDN-1425, RTN13-A x VDN-1606 and RTN13-A x VDN1608 showed high per cent of heterosis over check, which can be exploited on commercial basis.

\section{Introduction}

The heterosis has been now exploited self pollinated crops. It is well known that heterosis not only increases the yield but also provides a greater stability under varying environmental conditions. The $\mathrm{F}_{1}$ hybrid expresses considerably higher vigour over their superior parents. Heterosis breeding has been used as a potent genetic tool for exploiting the predominantly non- additive type of gene action, present in number of characters like seed yield now it is used in self pollinated crops like rice and wheat for increasing productivity of this major crop. Exploitation of heterosis or hybrid vigor is an important approach of crop improvement adopted in many of the crops all over the world. For exploitation of heterosis, choice of suitable parents is an important pre- requisite. 
The present investigation entitled "Heterosis studies in rice (Oryza sativa L.)" was undertaken with the objective to study the heterosis from three standard CMS lines and twelve restorer as parental material. Thirty six hybrids were developed by crossing three CMS and twelve restorer lines in $\mathrm{L} x \mathrm{~T}$ mating design.

The parents, hybrids and two standard checks were evaluated for nine quantitative characters, viz., days to 50 percent flowering, days to maturity, plant height at maturity, productive tillers per plant, tillers per square meter, thousand grain weight, grains per panicle, and yield per plant.

Heterosis was estimated as per Shull (1914) Heterosis is the superiority of $F_{1}$ over the mean of the parents or over the better parent or over the standard check (Hays et al., 1956), with respect to agriculturally useful traits. The primary objective of heterosis breeding is to achieve quantum jump in yield and quality aspects of crop plants.

In the present study, superiority of the hybrids was estimated over mid- parent, better parent and standard check (varietal and hybrid) for all the eight characters. The range of heterosis over mid-parent, better parent and standard checks and promising crosses with heterosis in respect of each of the character studied are presented in the Table1.

\section{Days to 50 per cent flowering (No.)}

From Table 1, it is quite evident that RTN13A x VDN1335RTN-17 A x VDN-1335, RTN17 A x VDN-1604 crosses showed significant negative heterosis over mid parent and better parent respectively and RTN17-A x VDN1335 showed significant negative heterosis over both the checks suggesting earliness of flowering. The hybrid RTN17-A x VDN1335 had highest negative heterobeltiosis value. The negative heterosis also substantiated the fact that the hybrids in general were early in flowering. The existence of both significant positive and negative heterotic effects over parents and checks suggests the presence of non-additive gene action for this trait. Sampath et al.,(1989), Ramlingam et al.,(1993), Manonmani and Ranganathan (1996) and Bhandarkar et al., (2005) have also reported earliness in rice hybrids.

\section{Days to maturity (No.)}

From Table 1, it is quite evident that cross RTN13-A x VDN1510, RTN-17 x VDN-1325 and RTN13-A x VDN1325 showed significant negative heterosis over mid parent and better parent, RTN17-A x VDN1335 showed significant negative heterosis over both the checks suggesting early maturity in hybrids. In general, the hybrids derived with RTN 13-A and RTN17-A as the female parent had highest negative heterosis over all the check hybrids.

The hybrids derived from these lines were observed to mature two to three days earlier than their parents. The existence of significant heterotic effect over parents and checks suggested the presence of non-additive gene action and dominance for this trait. Li et al., (2002), Bhave et al., (2002), Khrishna Veni et.al (2005) and Alam et al., (2004) have also reported early maturity in hybrids.

\section{Plant height (cm)}

In the present study, most of the hybrids exhibited heterosis in negative direction. From the table it is evident that, hybrids such as RTN13-A x VDN1335 and RTN-17 x VDN13336 recorded significant negative heterosis over mid parent, better parent and both standard checks indicating that experimental hybrids were dwarf than their parents and standard checks. 
Table.1 Heterosis for different eight characters over Mid Parent, Better Parent, standard check Variety and hybrid

\begin{tabular}{|c|c|c|c|c|c|c|c|c|c|c|c|c|c|}
\hline \multirow[t]{2}{*}{ S.N. } & \multirow[t]{2}{*}{ Crosses } & \multicolumn{3}{|c|}{ Days to $50 \%$ flowering } & \multicolumn{4}{|c|}{ Days to Maturity } & \multicolumn{5}{|c|}{ Plant Height } \\
\hline & & MP & BP & $\mathrm{CV}$ & SH & MP & $\mathrm{BP}$ & $\mathrm{CV}$ & SH & MP & BP & $\mathrm{CV}$ & SH \\
\hline 1 & IR 58525A *VDN1325 & $-2.18 *$ & $-7.24 * *$ & -1.10 & $-5.28 * *$ & -0.97 & $-4.01 * *$ & -1.10 & $-5.77 * *$ & $2.76^{*}$ & -0.87 & -1.71 & $-5.66 * *$ \\
\hline 2 & IR 58525A*VDN1336 & $8.21 * *$ & $4.48 * *$ & $12.65 * *$ & $6.70 * *$ & -0.94 & -1.34 & 1.65 & $-3.15^{*}$ & $15.74 * *$ & $10.3^{* *}$ & $12.18 * *$ & $7.67 * *$ \\
\hline 3 & IR 58525A*VDN1424 & 0.53 & -1.03 & $6.69 * *$ & 1.06 & $-4.97 * *$ & $-6.92 * *$ & 0.00 & $-4.72 * *$ & 1.87 & -1.08 & $-8.83 * *$ & $-12.50 * *$ \\
\hline 4 & IR 58525A*VDN1425 & -1.05 & -2.07 & $5.58 * *$ & 0.00 & $-3.48 *$ & $-6.95 * *$ & $3.31 *$ & -1.57 & 2.10 & 2.07 & $-5.94 * *$ & $-9.72 * *$ \\
\hline 5 & IR 58525A*VDN1335 & $-4.76 * *$ & $-10.3 * *$ & $-3.35^{*}$ & $-8.45 * *$ & $-4.95 * *$ & $-7.49 * *$ & $-4.68 * *$ & $-9.19 * *$ & 1.58 & $-7.6 * *$ & $4.02 * *$ & -0.16 \\
\hline 6 & IR 58525A*VDN1604 & $-5.74 * *$ & $-9.31 * *$ & -2.23 & $-7.39 * *$ & -1.70 & $-3.84 * *$ & $3.58 *$ & -1.31 & $4.93 * *$ & $2.37 * *$ & -0.83 & $-4.82 * *$ \\
\hline 7 & IR 58525A*VDN1606 & $-4.43 * *$ & $-6.90 * *$ & 0.38 & $-4.92 * *$ & $-4.52 * *$ & $-5.03 * *$ & -1.10 & $-5.77 * *$ & $4.75 * *$ & $2.21 *$ & -1.00 & $-4.98 * *$ \\
\hline 8 & IR 58525A*VDN1608 & $-5.78 * *$ & $-7.24 * *$ & 0.00 & $-5.28 * *$ & $-5.84 * *$ & $-8.56 * *$ & 0.00 & $-4.72 * *$ & $9.33 * *$ & $9.07 * *$ & 0.52 & $-3.53 * *$ \\
\hline 9 & IR 58525A*VDN1525 & $-3.29 *$ & $-3.79 *$ & $3.73 *$ & -1.75 & -1.83 & $-3.85^{*}$ & $3.31 *$ & -1.57 & $11.28 * *$ & $8.17 * *$ & -0.31 & $-4.32 * *$ \\
\hline 10 & IR 58525A*VDN1520 & $-5.45^{* *}$ & $-7.24 * *$ & 0.00 & $-5.28 * *$ & $-3.98 * *$ & $-4.74 * *$ & -0.28 & $-4.99 * *$ & $5.76 * *$ & $3.58 * *$ & $-4.54 * *$ & $-8.38 * *$ \\
\hline 11 & IR 58525A*VDN1510 & $-4.73 * *$ & $-6.21 * *$ & 1.12 & $-4.23 *$ & $-2.93 *$ & $-3.44 *$ & 0.55 & $-4.20 * *$ & $7.83 * *$ & $7.79 * *$ & -0.59 & $-4.59 * *$ \\
\hline 12 & IR 58525A*VDN1621 & -1.21 & -1.38 & $6.69 * *$ & 1.06 & -0.13 & $-3.95^{*}$ & $7.16^{* * *}$ & 2.10 & $6.70 * *$ & $4.26 * *$ & $-3.91 *$ & $-7.78 * *$ \\
\hline 13 & RTN 13A*VDN1325 & $-7.47 * *$ & $-12.1 * *$ & $-5.58 * *$ & $-10.6^{* *}$ & $-5.80 * *$ & $-10.51 * *$ & $-3.86^{*}$ & $-8.40 * *$ & $13.86^{* *}$ & $6.90 * *$ & $6.00 * *$ & 1.73 \\
\hline 14 & RTN 13A*VDN1336 & $-2.68 *$ & $-5.88 * *$ & 1.12 & $-4.23 *$ & -1.45 & $-3.85^{*}$ & $3.31 *$ & -1.57 & $-3.61 * *$ & $-10 . * *$ & $-9.05 * *$ & $-12.71 * *$ \\
\hline 15 & RTN 13A*VDN1424 & $-4.91 * *$ & $-6.23 * *$ & 0.75 & $-4.57 *$ & $-4.10 * *$ & $-4.10 * *$ & $3.03 *$ & -1.84 & $5.64 * *$ & $5.52 * *$ & $-8.17 * *$ & $-11.86^{* *}$ \\
\hline 16 & RTN 13A*VDN1425 & $-2.62 *$ & $-3.46^{*}$ & $3.73^{*}$ & -1.75 & -1.39 & -2.98 & $7.71 * *$ & 2.62 & $10.51 * *$ & $7.46 * *$ & -1.02 & $-5.00 * *$ \\
\hline 17 & RTN 13A*VDN1335 & $-7.16^{* *}$ & $-12.4 * *$ & $-5.95 * *$ & $-10.9 * *$ & $-4.84 * *$ & $-9.23 * *$ & -2.48 & $-7.09 * *$ & $17.33 * *$ & $3.99 * *$ & $17.14 * *$ & $12.43 * *$ \\
\hline 18 & RTN 13A*VDN1604 & $-8.08 * *$ & $-11.4 * *$ & $-4.83^{*}$ & $-9.86 * *$ & $-4.23 *$ & $-4.35 * *$ & $3.03 *$ & -1.84 & $10.89 * *$ & $5.26 * *$ & 1.97 & -2.13 \\
\hline 19 & RTN 13A*VDN1606 & $-3.90 * *$ & $-6.23 * *$ & 0.75 & $-4.57 * *$ & -2.34 & $-3.85^{*}$ & $3.31 *$ & -1.57 & $11.09 * *$ & $5.45^{* *}$ & 2.15 & -1.96 \\
\hline 20 & RTN 13A*VDN1608 & $-2.11 *$ & $-3.46^{*}$ & $3.73 *$ & -1.75 & -2.41 & $-3.28 *$ & $5.79 * *$ & 0.79 & $14.52 * *$ & $11.6 * *$ & 2.35 & -1.76 \\
\hline 21 & RTN 13A*VDN1525 & $-2.78 *$ & $-3.11 *$ & $4.09 *$ & -1.41 & $-3.59 *$ & $-3.59 *$ & $3.58 *$ & -1.31 & $11.58 * *$ & $11.5^{* *}$ & $-2.90 *$ & $-6.81 * *$ \\
\hline 22 & RTN 13A*VDN1520 & $-3.52 *$ & $-5.19 * *$ & 1.86 & $-3.52 *$ & -2.34 & $-3.59 *$ & $3.58 *$ & -1.31 & $4.49 * *$ & $3.71 *$ & $-8.37 * *$ & $-12.05 * *$ \\
\hline 23 & RTN $13 A^{*}$ VDN1510 & $-2.46^{*}$ & $-3.81 *$ & $3.35 *$ & -2.11 & $-5.99 * *$ & $-7.44 * *$ & -0.55 & $-5.25 * *$ & $6.17 * *$ & $3.18^{*}$ & $-4.84 * *$ & $-8.67 * *$ \\
\hline 24 & RTN 13A*VDN1621 & -0.35 & -0.69 & $7.44 * *$ & 1.76 & -0.13 & -1.98 & $9.37 * *$ & $4.20 * *$ & $15.13 * *$ & $14.5 * *$ & 0.73 & $-3.33^{*}$ \\
\hline 25 & RTN17 A*VDN1325 & $-5.38 * *$ & $-8.60 * *$ & $-5.20 * *$ & $-10.2 * *$ & $-3.23 *$ & $-4.70 * *$ & $-4.96 * *$ & $-9.45 * *$ & $2.52 *$ & $-2.80^{*}$ & $-3.62 *$ & $-7.50 * *$ \\
\hline 26 & RTN17 A*VDN1336 & -2.00 & $-3.58 *$ & 0.01 & $-5.27 * *$ & -2.05 & $-3.24^{*}$ & -1.10 & $-5.77 * *$ & $21.95 * *$ & $14.2 * *$ & $16.19 * *$ & $11.52 * *$ \\
\hline 27 & RTN17 A*VDN1424 & -0.71 & -1.07 & $3.35 *$ & -2.11 & 2.13 & -1.54 & $5.79 * *$ & 0.79 & $16.40 * *$ & $15.0 * *$ & 2.26 & -1.85 \\
\hline 28 & RTN17 A*VDN1425 & 0.53 & -0.35 & $5.21 * *$ & -0.35 & 1.96 & $-3.23^{*}$ & $7.44 * *$ & 2.36 & $12.73 * *$ & $10.7 * *$ & 2.00 & -2.10 \\
\hline 29 & RTN17 A*VDN1335 & $-6.54 * *$ & $-10.3 * *$ & $-7.06 * *$ & $-12.0 * *$ & $-3.63 *$ & $-4.70 * *$ & $-4.96 * *$ & $-9.45 * *$ & $4.99 * *$ & $-6.1 * *$ & $5.78 * *$ & 1.52 \\
\hline 30 & RTN17 A*VDN1604 & $-7.50 * *$ & $-9.32 * *$ & $-5.94 * *$ & $-10.9 * *$ & -0.40 & $-4.09 * *$ & $3.31 *$ & -1.57 & $10.12 * *$ & $5.57 * *$ & 2.27 & -1.84 \\
\hline 31 & RTN17 A*VDN1606 & $-2.89 *$ & $-3.58 *$ & 0.01 & $-5.27 * *$ & 1.35 & -0.79 & $3.31 *$ & -1.57 & $8.11^{* *}$ & $3.64 * *$ & 0.39 & $-3.64 * *$ \\
\hline 32 & RTN17 A*VDN1608 & $-3.21 *$ & $-3.56^{*}$ & 0.75 & $-4.57 * *$ & $-5.40 * *$ & $-9.57 * *$ & -1.10 & $-5.77 * *$ & $7.91 * *$ & $6.23 * *$ & $-2.57 *$ & $-6.49 * *$ \\
\hline 33 & RTN17 A*VDN1525 & -0.35 & -1.74 & $4.84 * *$ & -0.70 & -0.53 & $-4.10 * *$ & $3.03 *$ & -1.84 & $5.01 * *$ & $3.91 *$ & $-7.66^{* *}$ & $-11.37 * *$ \\
\hline 34 & RTN17 A*VDN1520 & -0.72 & -0.72 & $2.98 *$ & -2.46 & 0.81 & -1.58 & $3.03 *$ & -1.84 & $13.16^{* *}$ & $12.8 * *$ & 0.27 & $-3.76 * *$ \\
\hline 35 & RTN17 A*VDN1510 & -0.71 & -1.07 & $3.35 *$ & -2.11 & 1.35 & -0.79 & $3.31 *$ & -1.57 & $10.52 * *$ & $8.51 * *$ & 0.07 & $-3.95 * *$ \\
\hline 36 & RTN17 A*VDN1621 & & $-3.44 *$ & $4.47 * *$ & -1.05 & 0.13 & $-5.19 * *$ & $5.79 * *$ & 0.79 & $8.07 * *$ & $7.51 * *$ & $-4.46 * *$ & $-8.30 * *$ \\
\hline
\end{tabular}




\begin{tabular}{|c|c|c|c|c|c|c|c|c|c|c|c|c|c|}
\hline \multirow[t]{2}{*}{ S.N. } & \multirow[t]{2}{*}{ Crosses } & \multicolumn{12}{|c|}{ Productive tillers per plant (No.): Productive tillers/square meter (No.): Test Weight (g) } \\
\hline & & MP & BP & $\mathrm{CV}$ & SH & MP & BP & $\mathrm{CV}$ & SH & MP & BP & $\mathrm{CV}$ & SH \\
\hline 1 & IR $58525 \mathrm{~A} * \mathrm{VDN} 1325$ & $17.73 * *$ & $8.82 * *$ & $25.53 * *$ & $19.19 * *$ & $-93.7 * *$ & $-96.7 * *$ & $23.59 * *$ & $13.23 * *$ & $17 . .24 * *$ & $13.33 * *$ & $21.43 * *$ & $13.33 * *$ \\
\hline 2 & IR 58525A*VDN1336 & $25.88 * *$ & $22.27 * *$ & $19.70^{* *}$ & $13.66 * *$ & $-87.0 * *$ & $-93.3 * *$ & $13.57 * *$ & $4.05^{* *}$ & $18.51 * *$ & $6.66 * *$ & $14.29 * *$ & $6.67 * *$ \\
\hline 3 & IR 58525A*VDN1424 & $27.55^{* *}$ & $9.14 * *$ & $50.19 * *$ & $42.61 * *$ & $-93.4 * *$ & $-96.6 * *$ & $30.37 * *$ & $19.44 * *$ & $13.68 * *$ & $7.99 * *$ & $28.57 * *$ & $20.00 * *$ \\
\hline 4 & IR 58525A*VDN1425 & $24.87 * *$ & $7.92 * *$ & $45.03 * *$ & $37.71 * *$ & $-94.0 * *$ & $-96.9 * *$ & $17.37 * *$ & $7.53 * *$ & $8.89 * *$ & $8.89 * *$ & $16.67 * *$ & $8.89 * *$ \\
\hline 5 & IR 58525A*VDN1335 & $26.78^{* *}$ & $17.10^{* *}$ & $35.29 * *$ & $28.46 * *$ & $-94.3 * *$ & $-97.1 * *$ & $11.53^{* *}$ & 2.17 & $18.52 * *$ & $6.66^{* *}$ & $14.29 * *$ & $6.67 * *$ \\
\hline 6 & IR 58525A*VDN1604 & $15.80 * *$ & $15.03^{* *}$ & $12.61 * *$ & $6.93 * *$ & $-94.7 * *$ & $-97.3 * *$ & $3.87 * *$ & $-4.84 * *$ & $10.25 * *$ & $-4.45 * *$ & 2.38 & $-4.44 * *$ \\
\hline 7 & IR 58525A*VDN1606 & $9.72 * *$ & $6.64 * *$ & $4.40^{* *}$ & -0.87 & $-78.4 * *$ & $-88.9 * *$ & $-4.93 * *$ & $-12.90^{* *}$ & 0.00 & $-13.3 * *$ & $-7.14 * *$ & $-13.33^{* *}$ \\
\hline 8 & IR 58525A*VDN1608 & $7.08 * *$ & -1.00 & $14.15^{* *}$ & $8.39 * *$ & $-94.3 * *$ & $-97.0 * *$ & $11.85^{* *}$ & 2.47 & $5.62 * *$ & $4.44 * *$ & $11.91 * *$ & $4.45 * *$ \\
\hline 9 & IR 58525A*VDN1525 & $29.54 * *$ & $9.56 * *$ & $55.09 * *$ & $47.26 * *$ & $-94.9 * *$ & $-97.4 * *$ & -0.47 & $-8.82 * *$ & 0.00 & $-8.90 * *$ & -2.38 & $-8.89 * *$ \\
\hline 10 & IR $58525 A^{*}$ VDN1520 & $-15.9 * *$ & $-19.1 * *$ & $-14.28^{* *}$ & $-18.60^{* *}$ & $-94.7 * *$ & $-97.3 * *$ & $3.15^{*}$ & $-5.50 * *$ & 1.17 & $-4.45 * *$ & 2.38 & $-4.44 * *$ \\
\hline 11 & IR 58525A*VDN1510 & $8.71 * *$ & $7.89 * *$ & $7.24 * *$ & 1.82 & $-94.4 * *$ & $-97.1 * *$ & $8.59 * *$ & -0.52 & $17.07 * *$ & $6.66 * *$ & $14.29 * *$ & $6.67 * *$ \\
\hline 12 & IR 58525A*VDN1621 & $12.23 * *$ & $7.08 * *$ & $15.41 * *$ & $9.59 * *$ & $-95.0 * *$ & $-97.4 * *$ & -2.23 & $-10.43 * *$ & $2.54 *$ & $-11.1 * *$ & $-4.76 * *$ & $-11.11 * *$ \\
\hline 13 & RTN 13A*VDN1325 & $12.46^{* *}$ & $-2.43 *$ & $12.55^{* *}$ & $6.87 * *$ & $-4.33 * *$ & $-4.93 * *$ & -2.27 & $-10.47^{* * *}$ & -2.38 & -2.38 & -2.38 & $-8.89 * *$ \\
\hline 14 & RTN 13A*VDN1336 & $16.93 * *$ & $12.19 * *$ & $3.54 * *$ & -1.69 & $4.27 * *$ & $-3.68 * *$ & -2.23 & $-10.43^{* *}$ & $5.13^{* *}$ & -2.38 & -2.38 & $-8.89 * *$ \\
\hline 15 & RTN 13A*VDN1424 & $35.64 * *$ & $9.62 * *$ & $50.84 * *$ & $43.23 * *$ & $12.99 * *$ & $3.88 * *$ & $25.70 * *$ & $15.16^{* *}$ & $15 . .22 * *$ & $6.00 * *$ & $26.19 * *$ & $17.78 * *$ \\
\hline 16 & RTN 13A*VDN1425 & $31.79 * *$ & $7.48 * *$ & $44.44 * *$ & $37.15 * *$ & $16.20^{* *}$ & $12.10^{* *}$ & $22.42 * *$ & $12.15^{* * *}$ & $17 . .24^{* *}$ & $13.32 * *$ & $21.43^{* *}$ & $13.33^{* *}$ \\
\hline 17 & RTN 13A*VDN1335 & $24.82 * *$ & $8.22 * *$ & $25.04 * *$ & $18.73^{* *}$ & $14.05^{* *}$ & $6.27 * *$ & $7.87 * *$ & -1.18 & $15.39 * *$ & $7.14 * *$ & $7.14 * *$ & 0.00 \\
\hline 18 & RTN 13A*VDN1604 & $16.16^{* * *}$ & $9.07 * *$ & $5.36 * *$ & 0.04 & $15.92 * *$ & $4.07 * *$ & $5.63 * *$ & $-3.23 *$ & $17.33 * *$ & $4.76 * *$ & $4.76 * *$ & -2.22 \\
\hline 19 & RTN 13A*VDN1606 & $9.38 * *$ & $4.88 * *$ & $-3.08 * *$ & $-7.97 * *$ & $10.49 * *$ & -1.71 & -0.23 & $-8.60 * *$ & $12.00 * *$ & 0.00 & 0.00 & $-6.67 * *$ \\
\hline 20 & RTN 13A*VDN1608 & $23.37 * *$ & $7.06 * *$ & $23.44 * *$ & $17.21 * *$ & $3.93 * *$ & $-7.29 * *$ & $20.02 * *$ & $9.95 * *$ & $16.28 * *$ & $13.64 * *$ & $19.05^{* *}$ & $11.11^{* *}$ \\
\hline 21 & RTN 13A*VDN1525 & $33.79 * *$ & $6.97 * *$ & $51.42 * *$ & $43.77 * *$ & $6.25^{* *}$ & -0.56 & 0.94 & $-7.53 * *$ & $6.33 * *$ & 0.00 & 0.00 & $-6.67 * *$ \\
\hline 22 & RTN 13A*VDN1520 & $19.78 * *$ & $7.80 * *$ & $14.29 * *$ & $8.52 * *$ & $5.52 * *$ & 1.99 & $3.52 *$ & $-5.16 * *$ & $4.88 * *$ & 2.38 & 2.38 & $-4.44 * *$ \\
\hline 23 & RTN 13A*VDN1510 & $16.12 * *$ & $7.60 * *$ & $6.95 * *$ & 1.55 & $6.68 * *$ & 0.46 & 1.97 & $-6.58 * *$ & $6.33^{* *}$ & 0.00 & 0.00 & $-6.67 * *$ \\
\hline 24 & RTN 13A*VDN1621 & $15.74 * *$ & $3.41 * *$ & $11.45^{* *}$ & $5.83 * *$ & $13.95^{* *}$ & 1.76 & $3.29 *$ & $-5.37 * *$ & $11.98^{* * *}$ & 0.00 & 0.00 & $-6.67 * *$ \\
\hline 25 & RTN17 A*VDN1325 & $18.91 * *$ & 0.24 & $15.63 * *$ & $9.79 * *$ & $8.54 * *$ & $3.52 * *$ & $6.41 * *$ & -2.52 & $8.64 * *$ & $4.76^{* *}$ & $4.76 * *$ & -2.22 \\
\hline 26 & RTN17 A*VDN1336 & $14.38 * *$ & $6.23 * *$ & -1.96 & $-6.91 * *$ & $16.72 * *$ & $12.18 * *$ & $4.65^{* *}$ & $-4.13 * *$ & $17.33 * *$ & $12.82 * *$ & $4.76^{* *}$ & -2.22 \\
\hline 27 & RTN17 A*VDN1424 & $23.83 * *$ & $-2.48 *$ & $34.20 * *$ & $27.42 * *$ & $8.03 * *$ & $-4.35 * *$ & $15.75 * *$ & $6.05 * *$ & $7.86^{* *}$ & $-4.01 * *$ & $14.29 * *$ & $6.67 * *$ \\
\hline 28 & RTN17 A*VDN1425 & $28.62 * *$ & $2.18^{*}$ & $37.32 * *$ & $30.38 * *$ & $15.88^{* *}$ & $7.44 * *$ & $17.32 * *$ & $7.48 * *$ & $14.28 * *$ & $6.66 * *$ & $14.29 * *$ & $6.67 * *$ \\
\hline 29 & RTN17 A*VDN1335 & $26.56^{* *}$ & $6.63 * *$ & $23.20 * *$ & $16.98 * *$ & $14.82 * *$ & $11.35^{* *}$ & $3.87^{*}$ & $-4.84 * *$ & $22.67 * *$ & $17.95 * *$ & $9.52 * *$ & 2.22 \\
\hline 30 & RTN17 A*VDN1604 & $13.64 * *$ & $3.37 * *$ & -0.15 & $-5.19 * *$ & $17.83^{* *}$ & $9.91 * *$ & 2.54 & $-6.06^{* *}$ & $16.66^{* *}$ & $7.69 * *$ & 0.00 & $-6.67 * *$ \\
\hline 31 & RTN17 A*VDN1606 & $12.97 * *$ & $4.86^{* *}$ & $-3.10^{*}$ & $-7.99 * *$ & $16.44^{* *}$ & $7.57 * *$ & 0.35 & $-8.06^{* *}$ & $16.67 * *$ & $7.69 * *$ & 0.00 & $-6.67 * *$ \\
\hline 32 & RTN17 A*VDN1608 & $19.41 * *$ & 0.68 & $16.09 * *$ & $10.23^{* *}$ & $-5.79 * *$ & $-18.9 * *$ & $4.93 * *$ & $-3.87 *$ & $6.02 * *$ & 0.00 & $4.76^{* * *}$ & -2.22 \\
\hline 33 & RTN17 A*VDN1525 & $39.35 * *$ & $8.63 * *$ & $53.76^{* *}$ & $46.00^{* *}$ & $18.26^{* *}$ & $15.22 * *$ & $7.49 * *$ & -1.53 & $15.79 * *$ & $12.82 * *$ & $4.76^{* *}$ & -2.22 \\
\hline 34 & RTN17 A*VDN1520 & $20.23 * *$ & $4.99 * *$ & $11.31 * *$ & $5.69^{* *}$ & $12.25^{* *}$ & $11.40 * *$ & $5.51^{* *}$ & $-3.34 *$ & $8.86^{* *}$ & $7.50 * *$ & 2.38 & $-4.44 * *$ \\
\hline 35 & RTN17 A*VDN1510 & $21.59 * *$ & $9.20 * *$ & $8.53 * *$ & $3.05^{*}$ & $12.14 * *$ & $9.97 * *$ & 2.58 & $-6.02 * *$ & $10.53 * *$ & $7.69 * *$ & 0.00 & $-6.67 * *$ \\
\hline 36 & RTN17 A*VDN1621 & $18.97 * *$ & $3.16 * *$ & $11.19 * *$ & $5.58 * *$ & $17.54 * *$ & $9.03 * *$ & 1.71 & $-6.82 * *$ & $16.64 * *$ & $7.69 * *$ & 0.00 & $-6.67 * *$ \\
\hline
\end{tabular}




\begin{tabular}{|c|c|c|c|c|c|c|c|c|c|}
\hline \multirow[t]{2}{*}{ S.N. } & \multirow[t]{2}{*}{ Crosses } & \multicolumn{8}{|c|}{ No. of Grains per Plant (no) Yield Per plant (g) } \\
\hline & & MP & BP & $\mathrm{CV}$ & $\mathrm{SH}$ & MP & $\mathrm{BP}$ & $\mathrm{CV}$ & $\mathrm{SH}$ \\
\hline 1 & IR $58525 \mathrm{~A} * \mathrm{VDN} 1325$ & $9.26 * *$ & $7.87 * *$ & $8.33 * *$ & $4.86 * *$ & $10.75 * *$ & $10.64 * *$ & $10.59 * *$ & 1.88 \\
\hline 2 & IR 58525A*VDN1336 & $2.94 *$ & 0.97 & $2.74 *$ & -0.54 & $26.23 * *$ & $6.71 * *$ & $6.45 * *$ & -1.93 \\
\hline 3 & IR 58525A*VDN1424 & $-17.29 * *$ & $-36.74 * *$ & $-38.09 * *$ & $-40.07 * *$ & $9.45 * *$ & $7.70 * *$ & $10.99 * *$ & 2.24 \\
\hline 4 & IR 58525A*VDN1425 & $-17.57 * *$ & $-34.16 * *$ & $-35.56 * *$ & $-37.62 * *$ & $13.75 * *$ & $10.84 * *$ & $16.53 * *$ & $7.35 * *$ \\
\hline 5 & IR 58525A*VDN1335 & 0.56 & -1.77 & 0.81 & -2.42 & $-14.12 * *$ & $-14.14 * *$ & $-14.35^{* *}$ & $-21.09 * *$ \\
\hline 6 & IR 58525A*VDN1604 & $2.73 *$ & $-4.86 * *$ & $9.25 * *$ & $5.76 * *$ & $3.81 *$ & -2.27 & -2.50 & $-10.18 * *$ \\
\hline 7 & IR 58525A*VDN1606 & $6.81 * *$ & $-3.00 *$ & $16.30 * *$ & $12.58 * *$ & $12.66 * *$ & $12.50 * *$ & $12.24 * *$ & $3.39 *$ \\
\hline 8 & IR 58525A*VDN1608 & $10.79 * *$ & 1.63 & $19.16 * *$ & $15.35 * *$ & $3.65 *$ & $-3.20 *$ & $11.27 * *$ & 2.51 \\
\hline 9 & IR 58525A*VDN1525 & $-10.79 * *$ & $-27.23 * *$ & $-28.78 * *$ & $-31.06 * *$ & -0.84 & $-6.84 * *$ & $-7.07 * *$ & $-14.39 * *$ \\
\hline 10 & IR 58525A*VDN1520 & $2.81 *$ & $2.52 *$ & 0.91 & -2.32 & 0.92 & $-10.80 * *$ & $-11.01 * *$ & $-18.02 * *$ \\
\hline 11 & IR 58525A*VDN1510 & 2.11 & 1.58 & -0.59 & $-3.77 * *$ & $-6.08 * *$ & $-17.27 * *$ & $-17.47 * *$ & $-23.97 * *$ \\
\hline 12 & IR 58525A*VDN1621 & $4.95 * *$ & $3.14 * *$ & $4.55 * *$ & 1.21 & $4.80 * *$ & -0.34 & -0.57 & $-8.41 * *$ \\
\hline 13 & RTN 13A*VDN1325 & 2.11 & -2.33 & -1.91 & $-5.04 * *$ & $24.01 * *$ & $7.01 * *$ & $6.97 * *$ & -1.46 \\
\hline 14 & RTN 13A*VDN1336 & $11.07 * *$ & $5.58 * *$ & $7.44 * *$ & $4.01 * *$ & $22.47 * *$ & $19.39 * *$ & $-13.38 * *$ & $-20.20 * *$ \\
\hline 15 & RTN 13A*VDN1424 & $-22.76 * *$ & $-39.55 * *$ & $-44.57 * *$ & $-46.34 * *$ & $21.73 * *$ & $3.72 *$ & $6.88 * *$ & -1.54 \\
\hline 16 & RTN 13A*VDN1425 & $-20.93 * *$ & $-35.26 * *$ & $-40.63 * *$ & $-42.53 * *$ & $28.98 * *$ & $9.00 * *$ & $14.59 * *$ & $5.57 * *$ \\
\hline 17 & RTN 13A*VDN1335 & $4.88 * *$ & -0.70 & 1.91 & -1.35 & $25.18 * *$ & $8.13 * *$ & $7.83 * *$ & -0.67 \\
\hline 18 & RTN 13A*VDN1604 & $6.51 * *$ & $-4.22 * *$ & $9.99 * *$ & $6.47 * *$ & $23.00 * *$ & $12.16^{* *}$ & -1.21 & $-8.99 * *$ \\
\hline 19 & RTN 13A*VDN1606 & $16.73 * *$ & $3.00 *$ & $23.49 * *$ & $19.54 * *$ & $26.91 * *$ & $9.73 * *$ & $9.17 * *$ & $8.56 * *$ \\
\hline 20 & RTN 13A*VDN1608 & $7.45^{* *}$ & $-4.26 * *$ & $12.26 * *$ & $8.67 * *$ & $29.05 * *$ & $5.25^{*}$ & $20.99 * *$ & $11.45^{* *}$ \\
\hline 21 & RTN 13A*VDN1525 & $-11.99 * *$ & $-26.34 * *$ & $-32.45^{* *}$ & $-34.61 * *$ & $19.44 * *$ & $9.14 * *$ & $-4.30 *$ & $-11.84 * *$ \\
\hline 22 & RTN 13A*VDN1520 & $6.97 * *$ & $3.31 *$ & 1.69 & -1.57 & $18.37 * *$ & $15.24 * *$ & $-11.72 * *$ & $-18.67 * *$ \\
\hline 23 & RTN 13A*VDN1510 & $7.94 * *$ & $5.08 * *$ & 1.76 & -1.49 & $10.65 * *$ & $8.15^{* *}$ & $-17.82 * *$ & $-24.29 * *$ \\
\hline 24 & RTN 13A*VDN1621 & $8.91 * *$ & $3.72 *$ & $5.14 * *$ & 1.78 & $17.77 * *$ & $6.36 * *$ & $-4.29 * *$ & $-11.83 * *$ \\
\hline 25 & RTN17 A*VDN1325 & $3.89 *$ & 1.40 & 1.84 & -1.42 & $14.74 * *$ & $4.61 *$ & $4.57 * *$ & $-3.67 *$ \\
\hline 26 & RTN17 A*VDN1336 & 1.47 & -1.59 & 0.15 & $-3.06 * *$ & $18.93 * *$ & $9.24 * *$ & $-10.09 * *$ & $-17.17 * *$ \\
\hline 27 & RTN17 A*VDN1424 & $-22.35 * *$ & $-40.13 * *$ & $-42.74 * *$ & $-44.58 * *$ & $16.01 * *$ & $4.33^{*}$ & $7.52 * *$ & -0.95 \\
\hline 28 & RTN17 A*VDN1425 & $-24.14 * *$ & $-38.88 * *$ & $-41.56 * *$ & $-43.42 * *$ & $16.71 * *$ & $4.04 *$ & $9.39 * *$ & 0.77 \\
\hline 29 & RTN17 A*VDN1335 & -1.19 & $-4.56 * *$ & -2.06 & $-5.19 * *$ & $15.00 * *$ & $4.97 * *$ & $4.67 * *$ & $-3.57^{*}$ \\
\hline 30 & RTN17 A*VDN1604 & $7.52 * *$ & -1.47 & $13.14 * *$ & $9.52 * *$ & $17.77 * *$ & $13.91 * *$ & 0.34 & $-7.57 * *$ \\
\hline 31 & RTN17 A*VDN1606 & $6.94 * *$ & $-3.88 * *$ & $15.24 * *$ & $11.56 * *$ & $14.66 * *$ & $4.76^{* *}$ & $4.22 *$ & $-3.99 *$ \\
\hline 32 & RTN17 A*VDN1608 & $7.44 * *$ & -2.47 & $14.36 * *$ & $10.70 * *$ & $11.26 * *$ & $-4.54 * *$ & $9.74 * *$ & 1.09 \\
\hline 33 & RTN17 A*VDN1525 & $-27.89 * *$ & $-40.65 * *$ & $-43.25 * *$ & $-45.06 * *$ & $17.28 * *$ & $13.68 * *$ & -0.32 & $-8.17 * *$ \\
\hline 34 & RTN17 A*VDN1520 & $6.24 * *$ & $4.73 * *$ & $3.08^{*}$ & -0.21 & $4.83 * *$ & 1.20 & $-16.71 * *$ & $-23.27 * *$ \\
\hline 35 & RTN17 A*VDN1510 & 1.55 & 0.91 & -2.28 & $-5.40 * *$ & 1.74 & -2.16 & $-19.47 * *$ & $-25.81 * *$ \\
\hline 36 & RTN17 A*VDN1621 & $6.90 * *$ & $3.87 *$ & $5.29 * *$ & 1.92 & $7.46 * *$ & $2.88 *$ & $-7.42 * *$ & $-14.72 * *$ \\
\hline
\end{tabular}


This phenomenon was in agreement with reports of Alam et al., (2004), Li et al.,(2002) and Krishna veni et al., (2005). These dwarf hybrids probably may suit for mechanization and intercropping without much reduction in their yield levels

\section{Productive tillers per plant (No.)}

Productive tillers is one of the yield contributing character. In present investigation attempt has been made to establish relationship between productive tillers per plant and yield. For this the total number of panicle bearing tillers were counted. Hybrid exhibited intermediate expression for productive tillers per plant. However from the table it is observed that hybrid RTN17-A $\mathrm{x}$ VDN1525 recorded significant heterosis over mid parent and better parent. While IR58525A $x \quad$ VDN1335, RTN13A x VDN-1424, RTN13A x VDN-1525 and 13A x VDN-1425 recorded significant positive heterosis over varietal check and IR58525A x VDN1424 over hybrid checks. Similar results were observed by Sampath et al.,(1989) Krishna Veni et.al.(2005), Krishna Veni et al., (2005) and Bhandarkar et al.,(2005)

\section{Productive tillers per square meter (No.)}

Although there are many tillers per square meter, some of them does not contribute to yield. Therefore productive tillers per square meter form an important yield component that varies among genotypes. Among the experimental hybrids IR58525A x VDN1424 showed significant positive heterosis for productive tillers per square per meter over both checks and IRR-58025A x VDN-*1335 RTN13A $x$ VDN1424 showed significant positive heterobeltiosis, indicating the presence of non-additive and over dominance gene action. On contrary to this from the table The number of hybrids which recorded positive heterosis over mid parent, better parent, and varietal check Phule Samruddhi and hybrid checks Sahydri-3 were 32, 23, 23, and 13, respectively. Similar results were observed by Krishna Veni et al., (2005), Alam et al., (2004) and Yadav et al., (2004).

\section{Test weight (g)}

The thousand seeds weight of a genotype serves as an indicator of seed yield as it is an important character contributing to yield. In the present investigation, highly significant positive heterosis over mid-parent was recorded. However, majority of the hybrids recorded negative significant heterosis over the better-parent. From the table it is observed that hybrid RTN13-A x VDN1424 exhibited the highest mid-parent heterosis and heterosis over both checks. IR58525A x VDN1336 recorded highest heterosis over better parent. In general, the hybrid combinations with RTN13-A and IR58525-A as the female parent recorded highest positive heterotic values over the best check. This suggested the preponderance of dominant gene action in the determination of this trait. The significant positive heterosis for test weight is in conformity with the reports of $\mathrm{Li}$ et al., (2002), Singh et al., (2006), Singh et al., (2007).

\section{Number of grains per plant (No)}

Total number of seeds per plant is one of the important yield contributing character. In the present investigation attempt has been made to establish relationship between total number of grains per panicle, for this total number of grains per panicle were counted. Majority of hybrids recorded significant positive heterosis over best check indicates presence of nonadditive gene action. The hybrid IR-58025A x VDN1606, IIR-58025A $x$ VDN-1608 and RTN13A x VDN1606 exhibited significant positive heterosis over both the checks. Among the female parent, crosses involving 
IR58525A and RTN17-A exhibited higher heterosis over checks. The standard heterosis involving VDN1525 and VDN1336 as male parent was appreciably high than those involving other tester parents. The hybrid IR58525-A x VDN1525 displayed highest standard heterosis over standard checks compared to other crosses this cross combination worth for commercial exploitation after large scale evaluation over different environments. Similar results were reported by Veeresha et al., (2013) and Chamundeswari et al.,(2012).

\section{Yield per plant $(g)$}

A number of the research workers reported significant positive heterotic effects for seed yield. Seed yield per plant under well managed conditions would serve as a pointer to seed yield per unit area. Out of thirty six hybrids, thirty one hybrids recorded significant positive heterosis over mid parent. This suggests a strong influence of non additive gene action in determining seed yield per plant. Hybrids such as RTN13-A $\mathrm{x}$ VDN1608, RTN13-A x VDN1425 recorded positive heterosis over mid-parent and better parent, respectively while RTN13-A $\mathrm{x}$ VDN1608 and IR 2805A x VDN-1425 over standard checks. The predominance of nonadditive type of gene action for seed yield per plant favors for the development of potential high yielding hybrids. Similar conclusions were drawn by Sampath et al., (1989), Ramalingam et al., (1993), Manonmani and Ranganathan (1996), Chamundeswari et al.,(2012) and Sharma et al., (2013).

\section{References}

Alam, M. F., Khan, M. R., Nuruzzaman, M., Parvez, S., Swaraz, A. M., Alam, I. And Ahsan, N. 2004. Genetic basis of heterosis and inbreeding depression in Rice (Oryza sativa L.). J. Zhejiang
Univ. Sci., 5(4): 406- 411.

Bhandarkar, S., Rastogi, N. K. and Arvindkumar 2005. Study of heterosis in rice (Oryza sativa L.). Oryza, 42 (3) :218-219.

Bhave, S. G., Dhonukshe, B. L. and Bendale, V. W. (2002). Heterosis in hybrid rice. J. soil and crops, 12 (2): 183-186

Chamundeswari, N. Satyanarayana, P. V., Suryanarayana, Y. 2012. heterosis and combining ability studies for yield components in rice, Res.Crops; 2012. 13(3):1084-1089. 12 ref.

Hays, H. K., Immer, F. F. and Smith, D. L., 1956. Methods of Plant Breeding. McGraw Hill Book Publishing Company, Inc., New Delhi, pp. 21-34.

Krishana Veni, B., Shobha Rani, N. and Prasad, A. S. R. 2005. Heterosis for yield components and key quality traits basmati rice. Oryza,42 (2):97-102.

Li, W., Zhang, J. Z., Zhang, G. Q. and Zuo, Q. F. 2002. Analysis of Heterosis of Main Agronomic Traits in Indica-Japonica Lines of Rice. J. Southwest Agric. Univ., 24(4): 317-320.65.

Manonmani, S. and Ranganathan, T. B. 1996. Heterosis in early lines of indica rice. Madras Agric. J., 83 (9) : 548-551.

Ramalingam J., Vivekanandam, P., Vanniarajn, C. and Subbramanian, M. 1994. Heterosis in early rices. Ann. agric. Res., 15 (2):194-198.

Sampath, N., Rajeshekharan, S. and Vivekanandan, P. 1989. Heterosis in intervarital hybrids of rice (Oryza sativa L.). Madras Agric. J.,76 (9) : 507-511.

Sharma, S. K., Singh, S. K., Nandan, R., Amita Sharma, Ravindra Kumar, Kumar, V. and Singh, M. K. 2013. Estimation of heterosis and inbreeding depression for yield and yield related traits in rice (Oryza sativa L.). Molecular Plant Breeding; 2013:238246. 
Shull, G. H. 1914. Z. Inductive abstam. u. vere bungslehere, $12: 97-149$

Singh, R. V., Varma, O. P., Devivedi, J. L. and Singh, R. K. 2006. Heterosis studies in rice hybrids using CMSSystem. Oryza, 43(2): 154-156.

Singh, N. K., Singh, S., Singh, A. K., Sharma, C. L., Singh, P. K. and Singh, O. N. 2007. Study of heterosis in rice (Oryza sativa $\mathrm{L}$.) using line $\mathrm{x}$ tester mating system. Oryza, 44 (3): 260-263.

Veeresha, B. A., Salimath, P. M.,
Hanamaratti, N. G., Chetti, M. B., Nadaf, H. L. and Merwade, M. N. 2013.Combining ability analysis for yield and yield contributing traits in hybrid rice. Karnataka Journal of Agric.Sci. 26(4):494-496.

Yadav L. S., Maurya, D. M., Giri, S. P. and Singh, S. B. 2004. Nature and magnitude of heterosis for growth, yield and yield components in hybrid rice. Oryza, $41(1 \& 2): 1-3$.

\section{How to cite this article:}

Barhate, K. K., D. N. Borole and Kore, P. R. 2021. Heterosis for Yield and Yield Contributing Characters in Rice (Oryza sativa L.). Int.J.Curr.Microbiol.App.Sci. 10(06): 207-214. doi: https://doi.org/10.20546/ijcmas.2021.1006.022 